\title{
EL JUEGO
}

UN ASUNTO SERIO EN LA FORMACIÓN DE LOS NIÑOS Y LAS NIÑAS 



\section{EL JUEGO}

UN ASUNTO SERIO EN LA FORMACIÓN DE LOS NIÑOS Y LAS NIÑAS

Rita Flórez Romero

Jaime Castro Martínez

I. Ruth Camelo C.

Fernando Martelo Martínez

Irma Salazar Montenegro

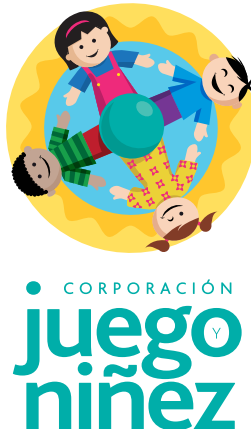




\section{POLI \\ POLITÉCNICO \\ GRANCOLOMBIANO \\ INSTITUCIÓN UNIVERSITARIA}

\section{(C) Institución Universitaria Politécnico Grancolombiano}

EL JUEGO: un asunto serio en la formación de los niños y las niñas

ISBN: 978-958-5544-22-2

E-ISBN: $978-958-5544-23-9$

Digital ISBN: $978-958-5544-24-6$

Octubre 2019

Editorial Politécnico

Grancolombiano

Calle 61 No. 7 - 66

Tel: 7455555, Ext. 1516

Bogotá, Colombia

\section{Autores}

Rita Flórez-Romero

Profesora Titular

Universidad Nacional de Colombia

\section{Jaime Castro-Martínez}

Docente Investigador

Institución Universitaria

Politécnico Grancolombiano

\section{Ruth Camelo C.}

Directora Ejecutiva

Corporación Juego y Niñez

\section{Fernando Martelo Martínez}

Presidente Consejo Fundadores

Corporación Juego y Niñez

Irma Salazar Montenegro

Gerente Técnica

Corporación Juego y Niñez

\section{Lider de publicaciones}

Eduardo Norman Acevedo

\section{Edición del Libro}

I.Ruth Camelo C. - Irma Salazar M.

Analista de Producción Editorial

Carlos Eduardo Daza Orozco

\section{Corrección de Estilo}

Hernán Dario Cadena

\section{Fotografías}

Patricia Arcos - Adriana Camelo Yuselfi Bermudez - Eliana Mejía Johana Méndez - Oscar Meneses Santiago Montaño - Irma Salazar Ricardo Salazar - Liseth Silva

Filiberto Yepes

\section{Diseño y Armada Electrónica}

Kilka Diseño Gráfico

\section{Impresión}

Xpress Estudio Gráfico

y Digital S.A.S

¿Cómo citar este libro?

Flórez-Romero, R., Castro-Martínez.

J., Camelo, R., Martelo Martínez,

F., \& Salazar Montenegro, I. (2019).

EL JUEGO: un asunto serio en la formación de los niños y las niñas (Vol. 1). Bogotá: Editorial Politécnico Grancolombiano.

\section{Creado en Colombia}

Todos los derechos reservados

No se permite la reproducción total o parcial de esta obra, ni su incorporación a un sistema informático, ni su tratamiento en cualquier forma o medio existentes o por existir, sin el permiso previo y por escrito de la Editorial de la Institución Universitaria Politécnico Grancolombiano

Para usos académicos y cientificos, la Institución Universitaria Politécnico Grancolombiano accede al licenciamiento Creative Commons del contenido de la obra con: Atribución - No comercial - Sin derivar - Compartir igual

El contenido de esta publicación se puede citar o reproducir con propósitos académicos siempre y cuando se dé la fuente o procedencia

Este libro es resultado de un proceso de investigación y ha sido evaluado por pares ciegos cumpliendo con los criterios de selectividad, temporalidad, normalidad y disponibilidad propuestos por COLCIENCIAS.
Grupos de investigación:

- Cognición y lenguaje en la infancia

- Psicología educación y cultura

Las opiniones expresadas son responsabilidad exclusiva del autor(es) y no constituye una postura institucional al respecto

La Editorial del Politécnico Grancolombiano pertenece a la Asociación de Editoriales Universitarias de Colombia (ASEUC) 


\section{Tabla de contenido}

\section{PRESENTACIÓN}

$--\cdot-\cdot-\cdot-$

¡Es hora de jugar!

CAPÍTULO 1

CAPITULO

El juego en el desarrollo infantil desde las ludotecas NAVES

20

\section{CAPÍTULO 2}

CAPITULO 2

La importancia de evaluar el impacto de las ludotecas en Colombia

CAPÍTULO 3

- - - - - - - -

Juego y ludotecas en el desarrollo de competencias ciudadanas,

emocionales y de creatividad en niños: estableciendo una línea base

CAPÍTULO 4

- - - - - - - -

El impacto de las ludotecas en el desarrollo de competencias ciudadanas, emocionales y de creatividad: hallazgos finales

CAPÍTULO 5

- - - - - - -

Conclusiones y Recomendaciones 



\section{¡Es hora de jugar!}

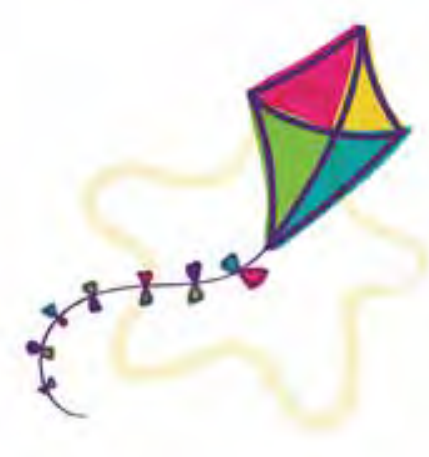

Existe una voluminosa bibliografía y testimonios de experiencias y prácticas acerca de las bondades del juego en el desarrollo humano que dicen, palabras más palabras menos, lo que afirma el presente libro -comprobado con investigación rigurosa de campo en nuestro país. 
Una lista de autores y trabajos académicos aparecerá en este libro, dándole base al marco conceptual sobre el cual se realizó esta primera investigación longitudinal en Colombia, sobre el impacto del juego jugado con Metodología Naves (niños, niñas aprendiendo,viviendo, experimentando y socializando), en seis ludotecas en seis municipios. Adicionalmente, tenemos a los autores e instituciones que vienen insistiendo cada día más en la importancia de las habilidades que se están atrofiando y que, por lo tanto, se necesitan desarrollar para vivir una vida plena en el mundo de hoy. Goleman las popularizó bajo el concepto de Inteligencia Emocional (2008), y subtituló su libro, "Por qué es más importante que el cociente intelectual". James Heckman ganó el premio Nobel de economía en el año 2000 por su trabajo, analizando datos de 40 años de estudios longitudinales en los Estados Unidos que lo llevaron a concluir que las habilidades blandas son tan o más importantes que el IQ para tener éxito en la vida, sobre todo cuando se forman desde edad temprana (Heckman, 2000). Kahneman, también premio nobel de economía, fue el primer psicólogo en ganar ese mismo premio lográndolo con sus estudios que resaltan la importancia de las emociones en el gobierno del pensamiento rápido (Sistema 1), en la toma de decisiones (Kahneman, 2012). Y esas son algunas de las habilidades que desarrolla el juego bien jugado.

Sabemos por todos ellos que el juego es:

“parte fundamental del ser humano, es una necesidad vital, que contribuye con el desarrollo de habilidades y potencialidades cognitivas, sociales, psicomotrices, emocionales, comunicativas, espirituales y estéticas; de la misma forma, transmite y transforma la cultura, permitiendo expresar, recrear y transformar la realidad de quienes lo viven, generando cambios en situaciones y comportamientos hacia la convivencia y la ciudadanía". (Salazar \& Flórez, 2019, p.62)

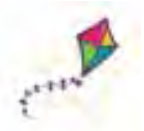


Francesco Tonucci (Fratto, 2015), nos viene diciendo, con ese tono afectuoso que le caracteriza, que el juego debe considerarse como un deber que hay que cumplir todos los días. Desde otra orilla, nos llama la atención que la Academia Americana de Pediatría esté recomendando a los pediatras recetar jornadas de juego diario. Así tal cual. En un reciente documento titulado El Poder del Juego (Yogman, Garner, Hutchinson, Hirsh-Pasek, \& Golinkoff, 2018), la academia muestra su preocupación con la disminución del tiempo que los niños dedican tanto en la escuela como en la vida hogareña a jugar a sus anchas. Y como demostración que el juego está en la agenda del día, en la versión 2019 del Foro Económico Mundial en Davos se lanzó la Real Play Coalition.

Martha Nussbaum (2014), entre sus Diez Capacidades Funcionales Humanas Centrales dedica una especialmente a la capacidad para "jugar y ser capaces de reír y disfrutar de actividades de ocio" (p.500). Y como derecho, el juego aparece en todas las legislaciones del mundo, empezando por la Declaración de los Derechos de los Niños de las Naciones Unidas.

Desde diferentes ópticas y en todos los continentes nos vienen reflexiones sobre el papel central que tiene el juego en nuestras vidas, llegándose incluso a necesitar revistas especializadas para su publicación, como por ejemplo, la American Journal of Play, que publica cuatro números al año desde 2008, y que hace parte de The Strong, el museo y biblioteca más grandes del mundo dedicados a la historia y la importancia del juego, bajo la inspiración de Brian Sutton-Smith (1924-2015), autor de La Ambigüedad del Juego (2009) y otros textos fundamentales.

Según el laureado biólogo Edward Wilson (1982), la creatividad es el rasgo que define nuestra especie y su objetivo definitivo es la autoconciencia, el poder entender quiénes somos y preguntarnos para dónde vamos. Así mismo, la evo- 
lución por selección natural de grupo estimuló la comunicación, el lenguaje, las historias y, por consiguiente, premió el desarrollo de la inteligencia emocional y el altruismo, gracias a los cuales sobrevivimos como especie. Otros han estudiado el juego en el comportamiento de los animales. Y estos son solo algunos. Algunos de los muchos y muchas que ven claramente la conexión juego - desarrollo humano - vida plena y que han encontrado inspiración en Huizinga (1972), y su clásico Hommo Ludens.

Sin embargo, no todas las corrientes pedagógicas le dan esa importancia al juego, porque ven al ser humano como un cajón vacío que se va llenando de repeticiones, de instrucciones e información. Y el juego implica libertad, exploración, imaginación, sentido de los otros jugadores, buen humor, manejo de situaciones difíciles y frustraciones, alegría de jugar. Actitud lúdica lo llaman algunos, a otros les daremos permiso de llamarlo "mamagalllismo", jugar a toda hora con las palabras y las situaciones. El mismo García Márquez, en su Proclama de los Sabios (2014/original publicado en 1994), nos lo dijo claro y directo:

\begin{abstract}
“Creemos que las condiciones están dadas como nunca para el cambio en nuestras sociedades, y que la educación será su órgano maestro. Una educación, desde la cuna hasta la tumba, inconforme y reflexiva, que nos inspire un nuevo modo de pensar y nos incite a descubrir quiénes somos en una sociedad que se quiera más a sí misma. Que aproveche al máximo nuestra creatividad inagotable y conciba una ética -y tal vez una estética- para nuestro afán desaforado y legítimo de superación personal". (p.24)
\end{abstract}

Él y los sabios recomendaron hacer de Colombia un país al alcance de los niños, porque un país así estará al alcance de todos para que todos alcancemos

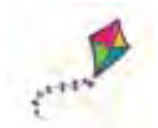


una vida plena. Un país donde la vida se viva en unos entornos que privilegian las habilidades socioemocionales y la creatividad.

Es tan importante llamar la atención sobre el asunto, que ha sido la misma OCDE (UNESCO-OCDE, 2016), la que ha tenido que preguntarse “¿Cuáles son las habilidades que impulsan el bienestar y el progreso social?" Al respecto, responde que:

“... ya es reconocida la necesidad de desarrollar un "niño completo", dotado de una serie equilibrada de habilidades cognitivas, sociales y emocionales que le permitan enfrentar mejor los desafíos del siglo XXI. Las habilidades sociales $\mathbf{y}$ emocionales no tienen un papel aislado: interactúan con las habilidades cognitivas, se estimulan unas a otras y mejoran aún más la probabilidad de que los niños consigan resultados positivos en el futuro". (p.11-12)

Y nos parece que aquí queda zanjada la cuestión.

Y de eso trata este libro. De presentar soporte con evidencia científica de lo que significa disfrutar del tiempo en las Ludotecas NAVES ${ }^{\circledR}$. Queremos que sea de gran utilidad para los que creen y cátedra de iniciación para quienes aún no lo entienden del todo.

Y no es este un tema solo teórico que puede admitir poca o mucha discusión, sino que esperamos que una vez comprendido se traduzca en acciones concretas en las poblaciones, en los colegios, en las políticas públicas que tienen que ver con desarrollo humano y social, que así deben ser todas. 
Y aquí pedimos permiso para hablar de nosotros y aclarar qué papel tenemos en todo esto. En la Corporación Juego y Niñez (CJN), tenemos esta pasión por el juego para contribuir a la vida en un mundo más lúdico y feliz, y por eso creamos en 1999 el Día de la Niñez y la Recreación, que se elevó como Ley de la República en diciembre de 2001. Todos los años mucha gente de nuestro país se prepara y dedica el mes de abril a recordarle al mundo que escuchen las voces que juegan cantando y que miren las manos que juegan pintando y pintan jugando, y participando llenan todo a su paso con su energía y buena onda.

La CJN tiene como propósito hacer transformación social desde el juego, considerado como lenguaje natural del ser humano, para lograr el desarrollo de las personas y sus comunidades. Para nosotros, los niños, niñas, adolescentes y jóvenes de Colombia se empoderan para influir en su realidad cuando fortalecen sus habilidades para la vida y su creatividad, jugando con una metodología basada en juego, científicamente probada, y por eso fue indispensable fomentar esta investigación con la Universidad Nacional de Colombia, porque la niñez en este país requiere que la dejen jugar y era necesario ratificar, con evidencia local, para convencer a los adultos que cuando jugamos nos conectamos con lo bueno, con lo mejor del ser humano. De esta manera concebimos que Colombia necesita avanzar en el desarrollo de las nuevas generaciones, y que el juego es el método ideal para su logro.

Esta fue la idea que nos motivó a crear e impulsar las ludotecas en Colombia en el año 2001. La ludoteca es un espacio de juego en donde las niñas y los niños desarrollan todo su potencial porque crean y recrean su cotidianidad, construyendo nuevos sentidos e incorporando reflexiones para generar nuevos aprendizajes, despliegan todo su potencial, viven una vida plena y mejoran su entorno. Para lograr esto es necesario formar cuidadores y propiciar entornos

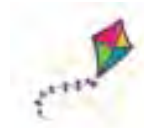


protectores de niñas, niños y adolescentes para que puedan desarrollar sus habilidades socioemocionales, su ciudadanía y su creatividad.

De este empeño nacen los y las ludotecarias, quienes vibran con la energía que tienen que canalizar todos los días. El ludotecario es un facilitador e inspirador del juego, inquieto y dinámico, quien dispone los recursos de la ludoteca para generar experiencias en las vidas de los participantes, aportando a la transformación de su ser, su hacer y su estar en el mundo.

Siempre nos han distinguido por nuestra atención al detalle, a la observación y la sistematización de experiencias y saberes, capacidad que se plasmó en la Metodología Naves ${ }^{\circledR}$ en 2008.

El trabajo de campo, y muchas veces en el campo, cerca de lo rural y lo urbano extremo, nos dio la garra suficiente para implementar un forma de administrar proyectos sociales, fundamentada en buenas prácticas gerenciales, con el acompañamiento de las personas de nuestro consejo directivo, conformado por líderes de empresas de primer nivel y del sector educativo.

Y lo más importante, la gente y el milagro del desarrollo humano y social. No poca cosa hemos aprendido de los niños y las niñas que nos han acogido y vienen a jugar con nosotros, lo que es un privilegio. $Y$ de sus familias. $Y$ de sus cuidadores. Y comprobamos a diario que también hay funcionarios que los respetan y se ocupan de sus cosas en sus corregimientos, municipios y gobernaciones.

Especial crédito debemos darle al papel que la academia colombiana ha tenido en el desarrollo de nuestras ideas, midiendo lo que hacemos en la práctica. En este caso, el Grupo de Investigación Cognición y Lenguaje en la Infancia 
y el Observatorio de Infancia, de la Universidad Nacional de Colombia, bajo la batuta de Rita Flórez-Romero y el Grupo de investigación Psicología educación y cultura con la participación del profesor Jaime Castro Martínez, investigador de la Institución Universitaria Politécnico Grancolombiano, quienes se midieron al reto de medir y probar lo que la intuición nos sugería. A ellos, nuestro reconocimiento por su dedicación e imparcialidad.

Queremos que Colombia sea un país que juegue. Un país que le sigue el juego a sus niños y niñas, adolescentes y jóvenes, porque "El juego es el recurso natural más importante en una economía creativa", tal como lo afirma Laura Seargeant Richardson (2011), Directora Creativa en la firma Argo Design, una firma global de innovación, quien ha llegado al extremo de afirmar que "en el futuro, las economías no serán impulsadas tanto por el capital financiero o incluso el capital científico, también lo serán por su capital de juego". Yo predigo que los países que toman el juego en serio, no exclusivamente nutriendo con él la educación, y a la fuerza de trabajo, sino también convirtiéndolo formalmente en un esfuerzo nacional, se destacarán y ascenderán en el orden mundial. ¿Será tan difícil de entender?

Por eso nos empeñamos en rescatar el juego para que con él los niños, niñas y adolescentes con sus familias transformen su mundo, y porque creemos en una sociedad donde haya más empatía, altruismo y en el que la gente se sienta contenta con lo que está viviendo. 


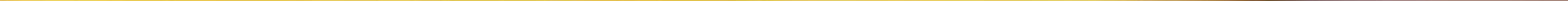

